\title{
COVID-19 pandemic effect on early pregnancy: are miscarriage rates altered, in asymptomatic women?
}

\author{
Keren Rotshenker-Olshinka ${ }^{1,2}$ (1) $\cdot$ Alexander Volodarsky-Perel ${ }^{1,3,4} \cdot$ Naama Steiner $^{1}$ - Eryn Rubenfeld ${ }^{1}$. \\ Michael H.Dahan ${ }^{1}$
}

Received: 31 August 2020 / Accepted: 14 October 2020 / Published online: 9 November 2020

○) Springer-Verlag GmbH Germany, part of Springer Nature 2020

\begin{abstract}
Purpose To evaluate the effect of the COVID-19 pandemic state on early, first-trimester pregnancies.

Methods A retrospective cohort study conducted at a university-affiliated fertility center in Montreal, Quebec, since the COVID-19 shut down, March 13 until May 6, 2020. Included: all women who came for a first-trimester viability scan during the study period (Study group) and between March 1, 2019 and May 17, 2019, approximately one year prior (Control). The study population denied symptoms of COVID-19.

We reviewed all first trimester scans. Early first-trimester pregnancy outcomes (Viable pregnancy, arrested pregnancy including biochemical pregnancy loss and miscarriage, and ectopic pregnancy) were measured as total number and percentage. A multivariate analysis was performed to control for other potentially significant variables, as was a power analysis supporting sample size.

Results 113 women came for a first-trimester viability scan in the study period, and 172 in the control period (5-11 weeks gestational age), mean maternal age $36.5 \pm 4.5$ and $37.2 \pm 5.4$ years $(p=0.28)$. Viable clinical pregnancy rate was not different between the two groups (76.1 vs. $80.2 \%$ in the pandemic and pre-pandemic groups $p=0.41)$. No significant difference was seen in the total number of arrested pregnancies (defined as the sum of biochemical, 1st trimester miscarriages, and blighted ova) (22.1 vs. $16.9 \% p=0.32)$, or in each type of miscarriage.

Conclusion The COVID-19 pandemic environment does not seem to affect early first-trimester miscarriage rates in asymptomatic patients.
\end{abstract}

Keywords COVID-19 $\cdot$ Pandemic $\cdot$ Early pregnancy $\cdot$ Miscarriage $\cdot$ First-trimester miscarriage

\section{Introduction}

COVID-19 is a respiratory illness caused by a new coronavirus-SARS-Cov-2 also known as COVID-19. It was first described December 2019 in Wuhan, China, and has since spread globally [1]. On March 11, 2020, the World HealthOrganization (WHO) declared a pandemic. As of early May 2020, there were over 60,000 confirmed cases in Canada, more than half of which were in Quebec [2] of which $49.1 \%$ occurred in Montreal[3].

The influence of the disease on pregnancy is not well understood. Current data from the American Centers for disease control (CDC) suggests that hospitalization rates

Keren Rotshenker-Olshinka

kerenlogic@gmail.com

Extended author information available on the last page of the article and ICU admissions in pregnant women diagnosed with COVID-19 are higher than in the non-pregnant population while mortality rates are similar [4]. There is conflicting evidence of vertical transmission [5, 6]. Evidence of coagulation disorders in COVID-19 patients are rising, which have the potential to adversely affect pregnancies [7, 8], particularly when combined with the inherent hypercoagulability of gestation.

The COVID-19 pandemic has medical implications reaching beyond the diagnosed patients. The community has self-isolated in the first few months of the pandemic, which comes with significant inherent stress and fear of both the unknown, significant disease, and noteworthy economic instability. These are unprecedented times and the influence of the pandemic environment, rather than the disease itself, on pregnancies is yet to be determined. When studying other extreme circumstances such as the exposure of 
pregnant women to armed conflict, a systematic review from 2017, suggest that pregnancy outcomes may be influenced by the associated chronic stress [9]. Two of the studies specifically addressing miscarriages - one analyzed the effect of in utero exposure to a maternal stressor in the form of the civil conflict in Nepal [10] and the other reported a connection between the preconception and pregnancy exposure to stress, as expressed by rocket-attack alarms, and an elevated risk of spontaneous abortions [11].

Another study on the impact of stress on the outcome of medically-assisted reproduction suggested that while stressors do not impact greatly on conception rates from medically-assisted reproduction, they may increase miscarriage rates [12].

In this study, we aimed to compare early pregnancy viability scans before and after the beginning of the COVID19 Pandemic. We aimed to investigate whether there is an adverse effect of the pandemic state on early pregnancies in terms of miscarriages.

\section{Materials and methods}

We conducted a retrospective cohort study. All women who came for a first-trimester viability scan at a university-affiliated fertility center in Montreal, Quebec, since the COVID19 shut down, March 13 until May 06, 2020 were included. The study population denied symptoms of COVID-19. For a control group, we reviewed all women who came for a first-trimester viability scan at the same center March 01, 2019 to May 17, 2019, approximately one year prior. The study was approved by the institutional review board (IRBnumber-2021-6705). All data were collected and entered by three authors (K.R.O., N.S., E.R.) and was reviewed again by the lead author (K.R.O) for verification.

We documented pregnancy outcomes as a viable clinical pregnancy when a fetal pole with a fetal heart rate was present. Multiple pregnancy occurred when two or more fetuses with a pulse were present. A biochemical pregnancy loss was determined in cases of a transient rise in beta-human chorionic gonadotropin ( $\beta$-hCG) without evidence of a gestational sac in-utero, and a subsequent fall in serum $\beta$-hCG levels. An ectopic pregnancy was determined in the presence of an extra-uterine gestational substance on pathology or with a high suspicion for an ectopic due to a $\beta$-hCG level above the threshold for ultrasound visibility with no evidence of an intrauterine pregnancy. Blighted ovum was defined as a gestational sac without a fetal pole via transvaginal ultrasound by $7-8$ weeks gestational age.

History of repeat pregnancy loss (RPL) was defined as two or more failed clinical pregnancies in accordance with the European Society of Human Reproduction and
Embryology (ESHRE) and the American Society for Reproductive Medicine (ASRM) guidelines [13, 14].

\section{Statistical analysis}

Data were analyzed using JMP Pro 14.1.0 software (SAS Institute Inc., USA). A data distribution was evaluated using the Shapiro-Wilk test in continuous variables. Normally distributed data were compared using Student's $t$ test and presented as means \pm standard deviations. Skewed data were presented as median (with interquartile range). The Mann-Whitney $U$ test was used for comparison in such cases. Categorical data were compared using the $\chi^{2}$ test and presented as a number of exposed cases and percentages of the total number of cases. A $p$ value $\leq 0.05$ was considered statistically significant.

To evaluate the association of the pandemic group and the adverse early pregnancy outcome controlling for other potentially significant variables we conducted a multivariate analysis. Only one of any highly correlated determinants was included in the model. Unadjusted and adjusted odds ratios (OR) with $95 \%$ confidence intervals (95\% CI) for study versus control group were calculated. We attempted to determine if the time of COVID-19 was a predictor of early pregnancy outcomes.

A power analysis revealed that a sample size of 58 patients in each group has a $90 \%$ power of showing a $15 \%$ difference in primary outcome with an alpha of $5 \%$.

\section{Results}

113 women came for a first-trimester viability scan in the study period, and 172 in the control period (5-11 weeks gestation).

The two groups were similar in demographic characteristics and fertility diagnosis (Table 1). Mean maternal age was $36.5 \pm 4.5$ and $37.2 \pm 5.4$ years in women coming for viability scans in the pandemic period and the pre-pandemic period respectively $(p=0.28)$. No significant differences were noted between the rate of RPL in the two groups (13.3 and $8.7 \%$ in the pandemic vs. pre-pandemic groups, $p=0.22$ ) nor was there a difference in their gestational history prior to pregnancy (median gravidity 1 in both groups, ranges $0-8$ and $0-9(p=0.13)$, median parity 1 , range $0-3$ in both groups $(p=0.31)$ (Table 1$)$.

Baseline serum thyroid stimulating hormone (TSH) levels were higher in the pre-pandemic group [1.3 (0.9-1.8) vs. 1.7 (1.2-2.3) IU/L, $p=0.001]$, although both groups fell in clinically normal ranges.

Method of conception; spontaneous (30.1 vs.31.4\%), IUI (30.1 vs. $29.7 \%$ ) or IVF (39.8 vs. $38.9 \%)$ were similar 
Archives of Gynecology and Obstetrics (2021) 303:839-845

841

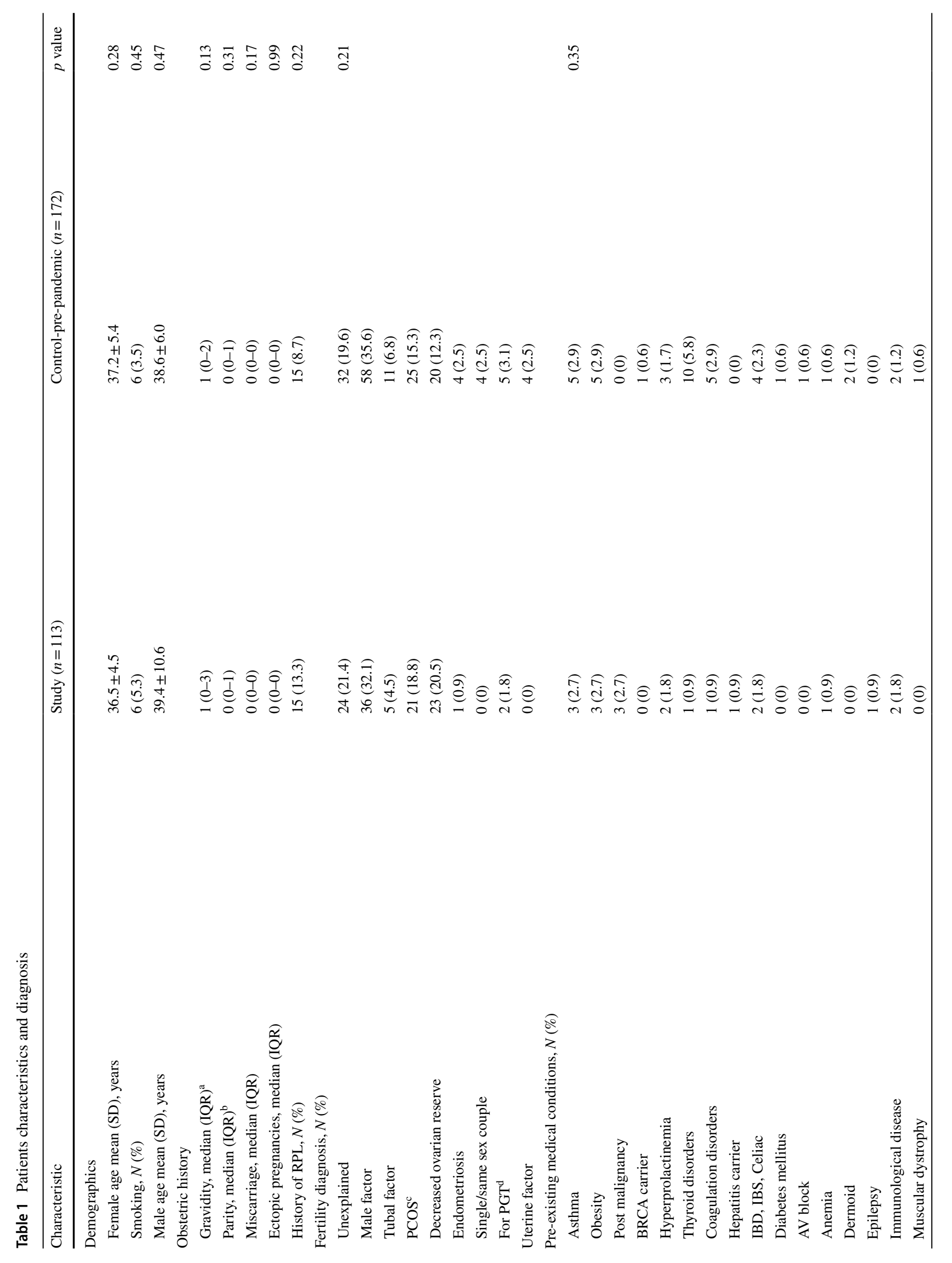

望 Springer 


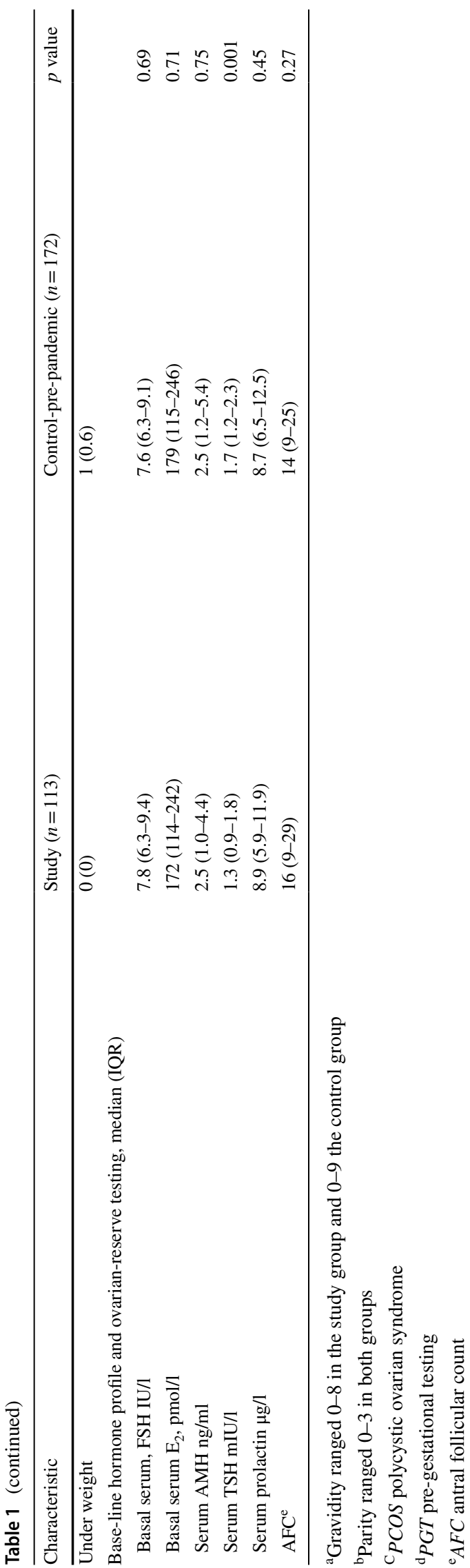

$(p=0.97)$, as was the use of ICSI (79.5 vs. $77.6 \% p=0.82)$. The use of donor sperm was less common in the pandemic group ( 1.8 vs. $9.9 \% p=0.01)$. Use of ovum donation was similar (18.2 vs. $17.9 \% ; p=0.97)$. All treatment characteristics are described in Table 2.

When reviewing pregnancy outcomes, the viable clinical pregnancy rate was not significantly different between the two groups (76.1 vs. $80.2 \%$ in the pandemic and prepandemic groups $p=0.41$ ). No significant difference was seen in the total number of arrested pregnancies (defined as the sum of biochemical, 1st trimester miscarriages, and blighted ova) ( 22.1 vs. $16.9 \% p=0.32$ ), Pregnancy outcomes are presented in Table 3.

Factors potentially associated with adverse early pregnancy outcome including female age, smoking, polycystic ovary syndrome (PCOS), obesity, hyperprolactinemia, coagulation disorders, serum TSH, antral follicular count (AFC), total motile sperm count (TMC) stage of embryo at transfer, frozen embryo transfers as well as the pandemic group were evaluated as possible confounders. The multivariate logistic regression model demonstrated that the pandemic group was not significantly associated with missed abortions, blighted ova, or biochemical pregnancies (Table 4) when controlling for confounding effects.

We then attempted to determine if the increased duration of stress may increase miscarriage rates with time during the pandemic. To do this we divided the study period into three duration groups: March 13-31st (42 patients), April 1-19th (36 patients), and April 20-May 6th (35 patients). We found no significant differences in rates of total arrested pregnancies (30.9 vs. 19.4 vs. $14.3 \% p=0.19$ ) between these periods. If anything miscarriage rates decreased with time although not statically so. As such, they did not demonstrate an effect of prolonged COVID-19 pandemic on increasing miscarriage rates.

\section{Discussion}

In our cohort, we assessed the influence of the pandemic environment, rather than the disease itself, on early pregnancy. We found no statistical difference in the number of arrested pregnancies between the COVID and pre-COVID periods. We were interested in assessing whether the stressrelated state of the pandemic would have an effect similar to that suggested when exposed to armed conflicts [9], or if possibly undetected asymptomatic infections may play a role.

Thirteen studies from different parts of the world that were previously analyzed demonstrated a significant relationship between armed conflict and increased rates of low birth rates [11]. Evidence also suggested a significant relationship with higher rates of low birth weights, stillbirth 
Table 2 Treatment and pregnancy characteristics

\begin{tabular}{|c|c|c|c|}
\hline Characteristic & Study $(n=113)$ & $\begin{array}{l}\text { Control-pre-pandemic } \\
(n=172)\end{array}$ & $p$ value \\
\hline \multicolumn{4}{|l|}{ Sperm parameters-current pregnancy ${ }^{a}$} \\
\hline $\mathrm{TMC}^{\mathrm{b}}$, median (IQR) & $18(6-43)$ & $12(5-47)$ & 0.23 \\
\hline Sperm donation, $N(\%)$ & $2(1.8)$ & $17(9.9)$ & 0.01 \\
\hline Surgically retrieved sperm, $N(\%)$ & $3(2.7)$ & $4(2.3)$ & 0.86 \\
\hline \multicolumn{4}{|l|}{ Pregnancy, $N(\%)$} \\
\hline Spontaneous & $34(30.1)$ & $54(31.4)$ & 0.97 \\
\hline IUI & $34(30.1)$ & $51(29.7)$ & \\
\hline IVF & $45(39.8)$ & $67(38.9)$ & \\
\hline ICSI (percentage of IVF patients) & $31(79.5)$ & $52(77.6)$ & 0.82 \\
\hline OD (percentage of IVF patients) & $8(18.2)$ & $12(17.9)$ & 0.97 \\
\hline \multicolumn{4}{|l|}{ Embryo stage at transfer, $N(\%)$} \\
\hline Cleavage-stage & $6(13.3)$ & $11(16.4)$ & 0.65 \\
\hline Blastocyst & $39(86.7)$ & $56(83.6)$ & \\
\hline Fresh embryo transfer, $N(\%)$ & $14(31.1)$ & $24(35.8)$ & 0.61 \\
\hline Frozen embryo transfers, $N(\%)$ & $31(68.9)$ & $43(64.2)$ & \\
\hline Embryos transferred, $N$. median (IQR) & $1(1-2)$ & $1(1-2)$ & 0.65 \\
\hline Progesterone supplementation, $N(\%)$ & $67(59.3)$ & $101(58.7)$ & 0.92 \\
\hline
\end{tabular}

${ }^{a}$ From IUI and IVF. Not applicable in spontaneous pregnancy

${ }^{\mathrm{b}} T M C$ total motile sperm count and prematurity [9]. One study reported a higher risk for miscarriage in women that were exposed to stress (measured as the mean number of rocket-attack alarms) during preconception and pregnancy with OR of 1.59 (95\% CI 1.13-2.03, $p=0.005$ ) [11]. This difference was attributed to the effect of stress on the pregnant individual, mediated by the rise in cortisol and a possible negative effect on the immune system $[12,15]$.

In our cohort, when comparing women who had their viability scans at the first months following the declaration of the pandemic with women in pre-pandemic similar months from the year prior, we demonstrate no significant difference in global or different types of miscarriage rates. The only differences seen in the baseline characteristics of the two groups were mean serum TSH levels, (fell in the normal range for both groups and is unlikely to be clinically relevant), and the use of donor sperm which was higher in the pre-pandemic period and may have favored lower miscarriage rates in the control group.

Not all subjects were followed throughout the first trimester. Therefore, the control group was selected for similar representation. In both groups, gestational age at last viability scan was similar (mean 7.1 and 6.6 weeks gestational age).

As for infection with COVID-19, we did not have any confirmed cases amongst our patients. Women coming to the clinic all denied symptoms of the disease. As our center is in Montreal which is the epicenter of the pandemic in Canada, we can assume some women were infected, although numbers would be small given our study group size.
The suggested mechanism of infection by the COVID-19 consists of the activation of angiotensin-converting enzyme receptor type 2 (ACE II), and the activation of the transmembrane protease, serine 2 (TMPRSS2) enzyme to cause the internalization of the virus $[16,17]$. It seems that both the ACE II receptor and the TMPRSS2 enzyme are required to be active for the cell to be infected. However, the TMPRSS2 enzyme may only be expressed after 24 weeks of pregnancy, and only in the extra-villous trophoblast, possibly limiting the timing of infections of the pregnancy tissue. In case there is an additional, unknown mechanism of the virus that could affect early pregnancies, we would have expected it to possibly have an adverse effect, favoring a higher miscarriage rate in the study group which was not demonstrated in our cohort. When addressing affected, symptomatic patients, it is possible that the hostile environment of an infected mother, rather than a direct infection of the fetus, can also cause adverse pregnancy outcomes, especially in the presence of low oxygenation saturation, high fever, or organ failure. No symptomatic patients were studied in this article.

We believe that a fertility center represented an optimal setting to study the effect of the pandemic milieu on miscarriage rates since almost all pregnant patients present for ultrasound evaluation at an early stage. This occurs because of the strong relationship with the doctor, the fear of miscarriages, and the elevated risk of ectopic pregnancy, related to fertility treatments and some of the underlying causes for infertility such as tubal factor. Even more so, during the pandemic since there was no other place to obtain these tests 
Table 3 Pregnancy outcomesfirst trimester

\begin{tabular}{llll}
\hline Pregnancy outcome & Study $(n=113)$ & $\begin{array}{l}\text { Control-pre-pandemic } \\
(n=172)\end{array}$ & $p$ value \\
\hline Gestational age at last scan median (IQR) & $7.1(6.5-8.1)$ & $6.6(6.2-8.0)$ & 0.07 \\
Viable clinical pregnancy, $N(\%)$ & $86(76.1)$ & $138(80.2)$ & 0.41 \\
Multiple pregnancy, $N(\%)$ & $4(3.5)$ & $8(4.6)$ & 0.65 \\
Arrested pregnancy-total $^{\mathrm{a}}, N(\%)$ & $25(22.1)$ & $29(16.9)$ & 0.32 \\
Biochemical pregnancy, $N(\%)$ & $4(3.5)$ & $3(1.7)$ & 0.34 \\
Miscarriage-1st trimester, $N(\%)$ & $16(14.2)$ & $22(12.8)$ & 0.76 \\
Blighted ovum, $N(\%)$ & $5(4.4)$ & $4(2.3)$ & 0.33 \\
Ectopic, $N(\%)$ & $1(0.9)$ & $4(2.3)$ & 0.36 \\
Molar pregnancy, $N(\%)$ & $1(0.9)$ & $1(0.6)$ & 0.76 \\
Cytotec use, $N(\%)$ & $11(37.9)$ & 0.86 \\
D\&C, $N(\%)^{\mathrm{b}}$ & $9(36)$ & $1(3.4)$ & 0.21 \\
Hematoma on ultrasound, $N(\%)$ & $3(12)$ & $10(5.8)$ & 0.33 \\
\hline
\end{tabular}

${ }^{\text {a }}$ Arrested pregnancy - total $=$ Biochemical +1 st trimester miscarriage + blighted ovum

${ }^{b}$ Percentage of arrested pregnancy-total
Table 4 Multivariate analysis of association between study group and adverse early pregnancy outcome

\begin{tabular}{|c|c|c|}
\hline \multirow[t]{2}{*}{ Parameter } & \multicolumn{2}{|c|}{ Study group versus control group } \\
\hline & $\begin{array}{l}\text { Unadjusted OR (95\% } \\
\text { CI) }\end{array}$ & Adjusted OR (95\% CI) \\
\hline Missed abortion & $1.3(0.7-2.1)$ & $1.2(0.5-5.2)$ \\
\hline $\begin{array}{l}\text { Biochemical preg- } \\
\text { nancy }\end{array}$ & $1.9(0.5-7.3)$ & $2.3(0.7-8.1)$ \\
\hline Blighted ovum & $2.1(0.5-8.7)$ & $2.0(0.5-8.5)$ \\
\hline
\end{tabular}

in the first trimester, due to the medical shut down. Moreover, our center is located in Montreal, which had the highest infection rate in Canada for COVID-19.

Although RPL rates did not statistically differ between the two groups, it was higher in the COVID-19 period group. This difference may have favored increased miscarriages in the pandemic period population, yet this did not occur, negating the influence of RPL.

Due to the retrospective character of this study, we couldn't have used tools to compare stress levels between the two groups. However, we believe the declaration of pandemic and its effect on almost every aspect of our lives, poses a unique stressor whose potential influence on early pregnancy we wished to explore.

In conclusion, the full effect of the pandemic on reproduction is yet to be determined. However, we demonstrate that in a non-symptomatic population, early first trimester pregnancies are not jeopardized. Evaluation of the continued effect of the pandemic environment on larger populations in the first trimester and later in pregnancy should be performed.
Author contributions K Rotshenker-Olshinka-Project development, Data collection and management, Manuscript writing and editing. A Volodarsky-Perel-Data analysis. N Steiner- Data collection and management. E Rubenfeld-Data collection and management. MH Dahan-Project development, Senior author, Manuscript editing.

Funding The study received no funding.

Availability of data and materials The data that support the findings of this study are available from the corresponding author, [K.R.O], upon reasonable request.

\section{Compliance with ethical standards}

Conflict of interest The authors declare that they have no conflict of interest.

Ethics approval The study was approved by the institutional review board (IRB-number-2021-6705).

Informed consent The study was approved by the McGill University Health Center (MUHC) institutional review board (Number 2021$6705)$ with a waiver of informed consent (Retrospective study).

\section{References}

1. Hui DS, Azhar EI, Madani TA, Ntoumi F, Kock R, Dar O et al (2020) The continuing 2019-nCoV epidemic threat of novel coronaviruses to global health-The latest 2019 novel coronavirus outbreak in Wuhan, China. Int J Infect Dis. 91:264-266

2. Coronavirus disease (COVID-19): Outbreak update [Internet]. Govermant of Canada. [cited 2020 May 5]. https://www.canad a.ca/en/public-health/services/diseases/2019-novel-coronaviru s-infection.html?topic $=$ tilelink

3. Situation of the coronavirus (COVID-19) in Québec [Internet]. Goverment of Quebec. 2020. https://www.quebec.ca/en/healt $\mathrm{h} /$ health-issues/a-z/2019-coronavirus/situation-coronavirus-inquebec/\#c63036 
4. Ellington S, Strid P, Tong VT et al (2020) Characteristics of Women of Reproductive Age with Laboratory-Confirmed SARSCoV-2 Infection by Pregnancy Status - United States. MMWR Morb Mortal Wkly Rep 69:769-775. https://doi.org/10.15585/ mmwr.mm6925a1

5. ACOG (American College of Obstetricians and Gynecologists) Practice advisory: novel coronavirus 2019 (COVID-19). Available at: https://www.acog.org/Clinical-Guidance-and-Publications/ Practice-Advisories/Practice-Advisory-Novel-Coronavirus2019. Retrieved 5 May 2020

6. RCOG (Royal College of Obstetricians and Gynaecologists) (2020) Royal College of Obstetricians and Gynaecologists. Coronavirus (COVID-19) Infection in Pregnancy. Version 7, pp 1-54

7. Fei Y, Tang N, Liu H, Cao W (2020) Coagulation Dysfunction A Hallmark in COVID-19. Arch Pathol Lab Med 144:1223-1229. https://doi.org/10.5858/arpa.2020-0324-SA

8. Levi M, Thachil J, Iba T, Levy JH (2020) Coagulation abnormalities and thrombosis in patients with COVID-19. Lancet Haematol 7(6): e438-e440

9. Keasley J, Blickwedel J, Quenby S (2017) Adverse effects of exposure to armed conflict on pregnancy: a systematic review, vol 2. BMJ Publishing Group, BMJ Global Health

10. Valente C (2015) Civil conflict, gender-specific fetal loss, and selection: a new test of the Trivers-Willard hypothesis. J Health Econ. 39:31-50. https://doi.org/10.1016/j.jhealeco.2014.10.005

11. Wainstock T, Lerner-Geva L, Glasser S, Shoham-Vardi I, Anteby EY (2013) Prenatal stress and risk of spontaneous abortion. Psychosom Med 75(3):228-235
12. Geisler M, Meaney S, Waterstone J, O’Donoghue K (2020) Stress and the impact on the outcome of medically assisted reproduction. Eur J Obstet Gynecol Reprod Biol 248:187-192

13. Bender Atik R, Christiansen OB, Elson J, Kolte AM, Lewis S, Middeldorp S et al (2018) ESHRE guideline: recurrent pregnancy loss. Hum Reprod Open. https://doi.org/10.1093/hropen/hoy00 4/4963604

14. The Practice Committee of the American Society for Reproductive Medicine (2012) Evaluation and treatment of recurrent pregnancy loss: a committee opinion. Fertil Steril 98:1103-1111. https://doi. org/10.1016/j.fertnstert.2012.06.048

15. Seng JS, Oakley DJ, Sampselle CM, Killion C, Graham-Bermann S, Liberzon I (2001) Posttraumatic stress disorder and pregnancy complications. Obstet Gynecol 97(1):17-22

16. D'Ardes D, Boccatonda A, Rossi I, Guagnano MT, Santilli F, Cipollone F, et al. COVID-19 and RAS: unravelling an unclear relationship. Int J Mol Sci. 2020; 21(8):3003. https://www.mdpi. com/1422-0067/21/8/3003

17. Li M, Chen L, Zhang J, Xiong C, Li X (2020) The SARSCoV-2 receptor ACE2 expression of maternal-fetal interface and fetal organs by single-cell transcriptome study. PLoS ONE 15(4):e0230295

Publisher's Note Springer Nature remains neutral with regard to jurisdictional claims in published maps and institutional affiliations.

\section{Affiliations}

\section{Keren Rotshenker-Olshinka ${ }^{1,2}$ - Alexander Volodarsky-Perel ${ }^{1,3,4} \cdot$ Naama Steiner $^{1} \cdot$ Eryn Rubenfeld $^{1} \cdot$ Michael H. Dahan ${ }^{1}$}

Alexander Volodarsky-Perel

alexander.volodarsky-perel@mail.mcgill.ca

Naama Steiner

steinern@bgu.ac.il

Eryn Rubenfeld

eryn.rubenfeld@mail.mcgill.ca

Michael H.Dahan

dahanhaim@hotmail.com

1 Division of Reproductive Endocrinology and Infertility,

Department of Obstetrics and Gynecology, McGill

University, Montréal, QC H3A 0G4, Canada
2 Department of Obstetrics and Gynecology Shaare Zedek Medical Center, Affiliated with the Hebrew University, 9103102 Jerusalem, Israel

3 Lady Davis Research Institute, Jewish General Hospital, Montréal, QC, Canada

4 Department of Obstetrics and Gynecology, Chaim Sheba Medical Center, Tel Hashomer, affiliated with the Sackler Faculty of Medicine, Tel Aviv University, Tel Aviv, Israel 